\title{
Desobstrução ineficaz das vias aéreas: mapeamento das atividades de enfermagem para crianças com infecção respiratória
}

\author{
I neffective airway clearance: plotting the nursing activities for children with respiratory \\ infection
}

\section{Limpieza ineficaz de las vías aéreas: mapeamiento de las actividades de enfermería para niños con infección respiratoria}

\author{
Flávia Paula Magalhães Monteiro', Viviane Martins da Silva", Marcos Venícios de Oliveira Lopes"', \\ Thelma Leite de Araujo ${ }^{\text {IV }}$
}

\begin{abstract}
RESUMO
As infecções respiratórias agudas são problemas que afetam crianças, especialmente, nos primeiros cinco anos de vida. Este estudo teve como objetivo comparar as intervenções de enfermagem desenvolvidas por enfermeiros assistenciais com aquelas preconizadas pela Nursing Intervention Classification ( NIC) para as crianças com até cinco anos de idade com doenças respiratórias agudas e que apresentam o diagnóstico de enfermagem Desobstrução Ineficaz de Vias Aéreas. Trata-se de um estudo exploratório-descritivo, com abordagem quantitativa, realizado na unidade de internação de um hospital pediátrico de Fortaleza - $C E$, no período de outubro a novembro de 2006. Foram entrevistadas 16 enfermeiras e listadas 48 atividades de enfermagem que caracterizaram cuidados específicos de enfermagem, dependentes da prescrição médica, interdependentes. Após essa etapa, foi realizado um comparativo com as atividades preconizadas pela NIC, no qual se observou atividades correspondentes e não-correspondentes. Foram 14 intervenções da NIC utilizadas no comparativo que somaram um total de 327 atividades. Para as intervenções de enfermagem NIC: Controle de vias aéreas e Monitorização respiratória houve um maior número de atividades correspondentes. Este estudo poderá trazer contribuições para a assistência de enfermagem às crianças com problemas respiratórios e que apresentam o diagnóstico de enfermagem Desobstrução ineficaz de vias aéreas.
\end{abstract}

Palavras chave: Diagnóstico de enfermagem; Criança hospitalizada; Cuidado da criança.

\section{ABSTRACT}

The acute respiration infections are problems which affect children, especially until five years old, presentation high numbers of obit in this age. This study has the objective to compare the interventions of nursing developed for assistants nurses with those presentations for Nursing Intervention Classification (NIC) for children until five years old with acute respiration infection and presentation the diagnosis of nursing Ineffective airway clearance. It is a study descriptive and quantitative performed in a pediatric hospital of Fortaleza-CE, in October and November of 2006. There were interviewed 16 nurses and listed 48 cares of nursing described in specifics cares of nursing, dependent of medical prescription, interdependent and related to problems respiratory of children. After that, there was a comparative with the activities of NIC, where observed correspondents and nocorrespondents activities. There were 14 interventions of nursing of NIC used in comparative which added up to 327 activities. For the interventions of nursing NIC: airway control and respiratory monitorization there were a bigger number of activities correspondents. This study could will to bring some contributions to the nursing assistance for children with respiratory problems and presentation the nursing diagnosis Ineffective airway clearance, like to offer a specific and qualified care.

Enfermeira. Bolsista de Apoio Técnico/aperfeiçoamento CNPq vinculada ao projeto de Pesquisa em Saúde Cardiovascular. Integrante do Grupo de Estudos em Diagnósticos, Intervenções e Resultados em Enfermagem (GEDIRE). Fortaleza/CE. E-mail: flaviapmm@yahoo.com.br.

"Enfermeira. Professora da Faculdade Católica Rainha do Sertão. Aluna do Curso de Doutorado em Enfermagem da UFC. E-mail: vivianemartinsdasilva@hotmail.com.

III Doutor em Enfermagem. Professor Adjunto do Departamento de Enfermagem da Universidade Federal de Ceará./ UFC. E-mail: marcos@ufc.br.

IV Doutora em Enfermagem. Professora Associada do Departamento de Enfermagem da Universidade Federal de Ceará/UFC. E-mail: thelmaarauj02003@yahoo.com.br. 
Monteiro FPM, Silva VM, Lopes MVO, Araújo TL. Desobstrução ineficaz das vias aéreas: mapeamento das atividades de enfermagem para crianças com infecção respiratória. Rev. Eletr. Enf. [Internet]. 2008;10(3):563-72. Available from: http://www.fen.ufg. br/ revista/v10/n3/v10n3a02.htm.

Key words: Nursing diagnosis; Child hospitalized; Child care.

\section{RESUMEN}

Las Infecciones respiratorias agudas son problemas que afectan niños, especialmente, en los primeros cinco años de vida. Este estudio tuvo como objetivo comparar las intervenciones de enfermería desarrolladas por enfermeros asistenciales con las preconizadas por la Nursing Intervention Classification (NIC) para los niños con hasta cinco años portadores de enfermedades respiratorias agudas y que presentan el diagnóstico de enfermería Limpieza Ineficaz de las Vías Aéreas. Se trata de un estudio exploratorio-descriptivo, con abordaje cuantitativo, realizado en la unidad de internación de un hospital pediátrico de Fortaleza - CE, en el período de octubre a noviembre de 2006. Fueron entrevistadas 16 enfermeras y enroladas 48 actividades de

\section{NTRODUÇÃO}

As Infecções Respiratórias Agudas (IRA) são problemas respiratórios que acometem crianças, especialmente, nos primeiros cinco anos de vida pela suscetibilidade e imaturidade do trato respiratório nessa faixa etária. Segundo Façanha e Pinheiro ${ }^{(1)}$ cerca de $40 \%$ das crianças que procuram atendimento médico estão acometidas por doenças respiratórias agudas, apresentando elevados índices de óbitos em crianças menores de cinco anos de idade. Além de sofrer a influência de fatores socioeconômicos, em virtude das desigualdades existentes, as IRA aumentam consideravelmente as taxas de hospitalização de crianças, elevando a demanda de serviços de saúde e exigindo uma complexidade de ações, em decorrência da peculiaridade da clientela assistida que, ainda em idade precoce, sofrem junto aos familiares, os processos traumáticos da internação hospitalar.

Essas doenças respiratórias acometem crianças com até cinco anos de idade e têm constituído a cada dia motivo de preocupação para os profissionais de saúde, dada a sua elevada morbidade, observada em termos mundiais, bem como a alta mortalidade que incide especialmente nos paises em desenvolvimento ${ }^{(2)}$. Para Oliveira, Dantas e Fonsêca $^{(3)}$, a maioria das crianças que adoece enfermería que caracterizaron cuidados específicos de enfermería, dependientes de la prescripción médica e interdependientes. Tras esa etapa, se ha realizado un comparativo con las actividades preconizadas por la NIC, en el cual se observó actividades correspondientes y no correspondientes. Se han identificado 14 intervenciones de la NIC utilizadas en el comparativo que sumaron un total de 327 actividades. Hubo un mayor número de actividades correspondientes para las intervenciones de enfermería NIC: Control de vías aéreas y Monitorización respiratoria. Este estudio podrá traer contribuciones para la asistencia de enfermería a los niños con problemas respiratorios y que presentan el diagnóstico de enfermería Limpieza ineficaz de las vías aéreas.

Palabras clave: Diagnósticos de enfermería; Niño ingresado; Cuidado del niño.

fica mais chorosa e dependente dos pais. Se houver a necessidade de uma hospitalização, seu quadro emocional tende a piorar, em função da possibilidade de afastar-se de casa e dos seus familiares, por conta do ambiente hospitalar a que será submetida. Durante o período de hospitalização, a criança fica restrita ao leito, cercada de pessoas estranhas e que, para ela, trazem mais dor e sofrimento. Esses fatores tornam-nas mais vulneráveis às conseqüências emocionais da hospitalização.

Em estudo realizado com crianças acometidas por IRA com ate cinco anos de vida, foram identificados os seguintes sintomas: tosse, dispnéia, coriza nasal, otalgia e chiado no peito $^{(4)}$. Essas doenças caracterizam-se por crises dependentes ou não da exposição da criança ao agente infeccioso, uma multiplicidade de formas clínicas e agentes etiológicos que deflagram episódios novos ou recidivantes.

De acordo com o ponto de vista operacional, as doenças respiratórias agudas são classificadas em doença muito grave, pneumonia grave, pneumonia e nãopneumonia ${ }^{(5)}$. Essa classificação varia de acordo com a idade da criança e as manifestações clínicas da doença.

Inúmeros fatores de risco estão relacionados aos problemas respiratórios em crianças, como idade, desnutrição, grau de 
Monteiro FPM, Silva VM, Lopes MVO, Araújo TL. Desobstrução ineficaz das vias aéreas: mapeamento das atividades de enfermagem para crianças com infecção respiratória. Rev. Eletr. Enf. [Internet]. 2008; 10(3):563-72. Available from: http://www.fen.ufg.br/revista/v10/n3/v10n3a02.htm.

escolaridade materna, desmame precoce, estação climática, tabagismo passivo. Estudos desenvolvidos nessa área mostram que o número de pessoas que coabitam a mesma casa está associado com o aumento dos problemas respiratórios, pois esta variável pode atuar como fator de risco pela maior possibilidade de transmissão de patógenos ${ }^{(6)}$.

Neste âmbito, a assistência à criança deve contemplar ações sistematizadas que englobam o levantamento de dados, o diagnóstico de enfermagem, a intervenção e a avaliação, funcionando como um todo integrado e interrelacionado. Torna-se necessário, então, traçar estratégias para o controle e uma avaliação mais específica e individualizada pelos profissionais de enfermagem através do uso do diagnóstico de enfermagem, onde o enfermeiro poderá atuar interpretando e julgando os problemas de saúde atuais ou potenciais baseados na manifestação das respostas do paciente.

Diagnóstico de enfermagem trata-se de um julgamento clínico sobre as respostas do paciente aos problemas de saúde reais ou potenciais ${ }^{(7)}$. Assim como os diagnósticos de enfermagem, as intervenções de enfermagem podem ser baseadas em um sistema de classificação como a Nursing Interventions Classification (NIC). Esta é composta por 30 classes, sete domínios, aproximadamente 500 intervenções compreendidas em principais, sugeridas e opcionais para cada diagnóstico, classificadas em níveis de importância e resolução do mesmo, e mais de 12.000 atividades $^{(8)}$.

As intervenções em enfermagem são definidas como qualquer ação com base no conhecimento científico, realizada para aumentar os resultados do cliente. Para isso, a NIC descreve atividades para serem realizadas com o propósito de implementar uma ou mais intervenção de enfermagem. Com vistas a fornecer um suporte às decisões clínicas e terapêuticas e constituir uma ferramenta ao cuidado às crianças acometidas por doença respiratória aguda e que apresentam diagnósticos de enfermagem associados ao sistema respiratório, esse estudo teve como objetivo comparar as intervenções de enfermagem desenvolvidas por enfermeiros de um hospital pediátrico de Fortaleza-Ce com aquelas apresentadas pela Nursing Intervention Classification (NIC) para as crianças com até cinco anos de idade acometidas por doença respiratória aguda, que apresentam o diagnóstico de enfermagem Desobstrução Ineficaz de Vias Aéreas.

\section{MÉTODOS}

Trata-se de um estudo do tipo exploratório-descritivo com abordagem quantitativa, realizado em um hospital pediátrico da rede pública do município de Fortaleza-Ceará, no mês de outubro de 2006. Neste mesmo período, foi realizado um levantamento das atividades de enfermagem indicadas pelas enfermeiras assistenciais que prestavam o cuidado às crianças na unidade da internação de um hospital pediátrico para fazer um comparativo entre as atividades desenvolvidas pelas enfermeiras com aquelas preconizadas pela NIC. A instituição, local de coleta de dados, desse estudo atende crianças de baixo nível sócio-econômico, cujas patologias mais comuns são diarréias, infecções respiratórias agudas e asma. O serviço de saúde é composto por atendimento ambulatorial, emergência e internação.

A população desta etapa foi constituída por enfermeiras com atuação na área assistencial da unidade de internação de um hospital pediátrico do município de FortalezaCeará, que atende a clientela com alterações respiratórias em situações clínicas ou de emergência. Os participantes do estudo foram selecionados pela escala mensal de enfermeiros da unidade hospitalar no período da coleta de dados que atuavam na assistência direta às crianças internadas com IRA.

A amostra foi composta por um total de 16 enfermeiras. As atividades prescritas pelas enfermeiras foram submetidas a um comparativo com as atividades preconizadas pela NIC para o diagnóstico de enfermagem em estudo. Foram 14 intervenções de enfermagem selecionadas de acordo com as atividades descritas pelas enfermeiras, distribuídas em três intervenções prioritárias, sete intervenções sugeridas e quatro intervenções optativas para 
Monteiro FPM, Silva VM, Lopes MVO, Araújo TL. Desobstrução ineficaz das vias aéreas: mapeamento das atividades de enfermagem para crianças com infecção respiratória. Rev. Eletr. Enf. [Internet]. 2008; 10(3):563-72. Available from: http://www.fen.ufg. br/revista/v10/n3/v10n3a02.htm.

o diagnóstico Desobstrução ineficaz de vias aéreas. Foi utilizado um instrumento do tipo formulário, contendo dados relativos às atividades de enfermagem indicadas pelas enfermeiras às crianças que apresentavam o diagnóstico de enfermagem Desobstrução ineficaz de vias aéreas. Para facilitar a listagem das atividades desenvolvidas por elas, foram descritas as características definidoras do diagnóstico, consideradas prevalentes em estudo similar para o mesmo diagnóstico de enfermagem em questão(9). A comparação das atividades indicadas pelas enfermeiras com as atividades preconizadas pela NIC para o diagnóstico de enfermagem Desobstrução ineficaz de vias aéreas foi feita através da construção de um quadro. Para a comparação das intervenções prescritas foi realizada uma análise geral de todas as intervenções propostas pela NIC para o diagnóstico de enfermagem Desobstrução ineficaz de vias aéreas. As intervenções da NIC foram transcritas em uma coluna e na outra coluna as atividades de enfermagem prescritas pelas enfermeiras assistenciais, consideradas correspondentes. Uma vez identificadas as intervenções pelo título, analisamos as atividades indicadas pelas enfermeiras e consideramos correspondentes as atividades preconizadas pela NIC, buscando identificar palavras diferentes com significados semelhantes, método utilizado por Napoleão(10) em estudo similar. A proposta de estudo foi encaminhada ao Comitê de Ética em Pesquisa, procurando atender aos aspectos contidos na resolução 196/96 sobre pesquisa envolvendo seres humanos do Conselho Nacional de Saúde/Ministério da Saúde ${ }^{(11)}$, tendo sido aprovada sob o número 165/06.

\section{RESULTADOS E DI SCUSSÃO}

Foram listadas 48 atividades de enfermagem diferentes que são desenvolvidas pelas 16 enfermeiras assistenciais durante suas atividades diárias na unidade de internação pediátrica. Para listar essas atividades, as enfermeiras descreveram ações já desenvolvidas por elas no cotidiano, sem consultar a literatura específica.
Estas atividades foram então comparadas com as 14 intervenções e as 327 atividades propostas pela NIC. Verificou-se, então, correspondência entre 63 atividades.

As intervenções de enfermagem NIC com maior número de correspondências foram: Controle de vias aéreas e Monitorização respiratória. Ressalta-se que estas intervenções são classificadas pela NIC como prioritárias para o diagnóstico em questão (Tabelas 1 e 2). O fato de o estudo destacar a assistência de crianças com o diagnóstico de Desobstrução ineficaz de vias aéreas, provavelmente justifica serem essas as intervenções mais citadas. 


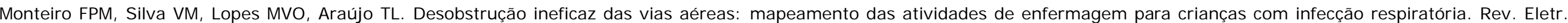
Enf. [Internet]. 2008;10(3):563-72. Available from: http://www.fen.ufg. br/revista/v10/n3/v10n3a02.htm.

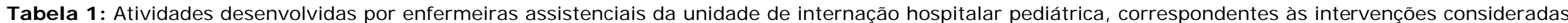
prioritárias preconizadas pela NIC. Fortaleza, 2006

\section{Atividades propostas pela NIC}

\begin{tabular}{|c|c|}
\hline Atividades propostas pela NIC & Atividades desenvolvidas pelas enfermeiras \\
\hline Realizar fisioterapia do tórax, conforme adequado ${ }^{1}$ & Realizar tapotagem; Solicitação da avaliação do fisioterapeuta. \\
\hline Remover secreções, encorajando o tossir ou aspirando ${ }^{1}$ & Aspiração das vias aéreas superiores, s/n; Estimular a tosse. \\
\hline Administrar tratamentos com aerosol, conforme adequado ${ }^{1}$ & Aerossolterapia CPM. \\
\hline Regular a ingestão de líquidos para otimizar o equilíbrio de líquidos ${ }^{1}$ & $\begin{array}{c}\text { Estimular ingesta hídrica e/ ou líquido; Orientar a mãe/acompanhante para estimular } \\
\text { a ingesta líquida; Administrar hidratação venosa CPM. }\end{array}$ \\
\hline Posicionar a criança para maximizar o potencial ventilatório ${ }^{1,8}$ & $\begin{array}{c}\text { Elevar a cabeceira do leito; Favorecer a expansão torácica, com mudança de } \\
\text { decúbito; Incentivar à mudança de decúbito. }\end{array}$ \\
\hline Encorajar a respiração lenta e profunda, o virar e o tossir ${ }^{1,8}$ & $\begin{array}{l}\text { Incentivar à mudança de decúbito; Estimular a tosse; Estimular a respiração } \\
\text { profunda. }\end{array}$ \\
\hline $\begin{array}{c}\text { Auscultar os sons respiratórios, observando áreas de ventilação diminuídas ou } \\
\text { ausentes e a presença de ruídos adventícios }\end{array}$ & Realizar avaliação pulmonar (ausculta); Realizar exame físico; \\
\hline Administrar broncodilatadores, conforme adequado ${ }^{1,8}$ & Administração de medicamentos CPM; Aerossolterapia CPM. \\
\hline Monitorar o estado respiratório e a oxigenação, conforme apropriado ${ }^{1,8}$ & $\begin{array}{l}\text { Monitorização/verificação dos sinais vitais; Monitorização com oximetria de } \\
\text { pulso; Observação do padrão respiratório da criança. }\end{array}$ \\
\hline Administrar ar umidificado ou oxigênio, conforme adequado ${ }^{1,4,8}$ & Oxigenoterapia CPM; Aerossolterapia CPM. \\
\hline Posicionar de modo a aliviar a dispnéia 1, 7,8 & $\begin{array}{l}\text { Elevar a cabeceira do leito; Favorecer a expansão torácica, com mudança de } \\
\text { decúbito. }\end{array}$ \\
\hline Monitorizar ruídos respiratórios, tais como sibilos e roncos ${ }^{2}$ & Avaliação pulmonar (ausculta); Realizar exame físico. \\
\hline Monitorizar aumento de agitação, ansiedade e falta de $\operatorname{ar}^{2}$ & $\begin{array}{l}\text { Observação do padrão respiratório da criança; Monitorização/verificação dos sinais } \\
\text { vitais; Monitorização com oximetria de pulso; Encorajar à criança ao repouso no } \\
\text { leito; I dentificar as causas da agitação; Observar nível de consciência da } \\
\text { criança; Avaliar a perfusão periférica; Colheita de exame para gasometria. }\end{array}$ \\
\hline Monitorar dispnéia e eventos que possam aumentá-la ou piorá-la² & $\begin{array}{l}\text { Monitorização/verificação dos sinais vitais; Observação do padrão respiratório da } \\
\text { criança; Monitorização com oximetria de pulso. }\end{array}$ \\
\hline Monitorizar presença de crepitações, se apropriado² & Avaliação pulmonar (ausculta). \\
\hline Iniciar tratamentos de fisioterapia respiratória (nebulização, $\mathrm{s} / \mathrm{n})^{2}$ & Aerossolterapia CPM. \\
\hline Percutir tórax anterior e posterior, dos ápices para as bases, bilateralmente ${ }^{2}$ & Realizar exame físico; \\
\hline $\begin{array}{c}\text { Anotar movimentos do tórax, observando simetria, uso de músculos acessórios } \\
\text { e retrações musculares, supraclaviculares e intercostais }{ }^{2}\end{array}$ & Realizar exame físico; \\
\hline Monitorizar padrões respiratórios: bradpnéia, taquipnéia, hiperventilação, etc ${ }^{2}$ & $\begin{array}{c}\text { Monitorização/verificação dos sinais vitais; Observação do padrão respiratório da } \\
\text { criança. }\end{array}$ \\
\hline Palpar expansibilidade pulmonar ${ }^{2}$ & Realizar exame físico. \\
\hline Observar início, característica e duração da tosse ${ }^{2}$ & Observar consistência, característica e quantidade de secreção. \\
\hline Monitorar a freqüência, ritmo, profundidade e esforço das respirações ${ }^{2,5}$ & $\begin{array}{l}\text { Monitorização/verificação dos sinais vitais; Observação do padrão respiratório da } \\
\text { criança. }\end{array}$ \\
\hline $\begin{array}{c}\text { Determinar a necessidade de aspiração através da ausculta de estertores e } \\
\text { roncos nas vias aéreas }{ }^{2,6}\end{array}$ & Aspiração das vias aéreas superiores. \\
\hline Auscultar sons pulmonares após tratamentos e anotar resultados ${ }^{2,6}$ & Avaliação pulmonar (ausculta). \\
\hline
\end{tabular}




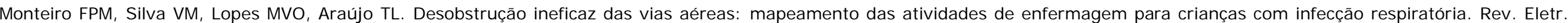
Enf. [Internet]. 2008;10(3):563-72. Available from: http://www.fen.ufg.br/revista/v10/n3/v10n3a02.htm.

\begin{tabular}{|c|c|}
\hline Atividades propostas pela NIC & Atividades desenvolvidas pelas enfermeiras \\
\hline Monitorizar secreções respiratórias da criança ${ }^{2,6}$ & $\begin{array}{c}\text { Observar consistência, característica e quantidade de secreção; Observar presença } \\
\text { de secreções nasais e orais. }\end{array}$ \\
\hline $\begin{array}{c}\text { Auscultar sons respiratórios, observando áreas de ventilação diminuída e, } \\
\text { presença de ruídos adventícios }{ }^{2,8}\end{array}$ & Avaliação pulmonar (ausculta); Realizar exame físico. \\
\hline Encorajar a criança a fazer várias respirações profundas ${ }^{3,8}$ & Estimular respiração profunda; \\
\hline Promover a hidratação sistêmica com líquidos, & ação venosa CPM; Estimular aum \\
\hline
\end{tabular}

Promover a hidratação sistêmica com líquidos, conforme apropriado ${ }^{3,10}$ líquidos; Orientar à mãe/acompanhante para aumentar a ingesta de líquidos

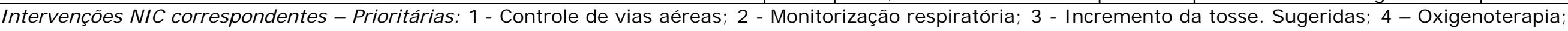

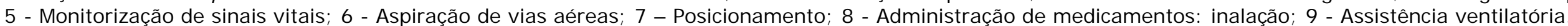
Optativas: 10 - Controle de líquidos.

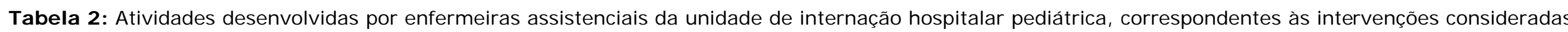
sugeridas e optativas preconizadas pela NIC. Fortaleza, 2006

\section{Atividades propostas pela NIC}

Limpar secreções orais, nasais e traqueais, quando adequado ${ }^{1}$

Manter vias aéreas desobstruídas ${ }^{1,6}$

Monitorar a eficácia da terapia com o oxigênio, quando adequado ${ }^{1}$ Monitorar a ansiedade da criança relacionada à necessidade de terapia com oxigênio ${ }^{1}$

Observar os sinais de hipoventilação induzida pelo oxigênio ${ }^{1}$

Ajustar o equipamento de oxigênio e administrar por meio de um sistema aquecido e umidificado ${ }^{1}$

Monitorar a pressão arterial, o pulso, a temperatura e o padrão respiratório quando adequado $2,7,12$

Monitorar e relatar os sinais de hipotermia e hipertermia ${ }^{2}$ Monitorar os batimentos cardíacos ${ }^{2}$

Monitorar a freqüência e o ritmo respiratório ${ }^{2}$

Monitorar os sons pulmonares ${ }^{2,4}$

Monitorar os padrões respiratórios anormais ${ }^{2,6}$

Monitorar a oximetria de pulso ${ }^{2,6}$

Iniciar e manter uma monitoração contínua da temperatura com aparelho, quando adequado

Monitorar a cor, a temperatura e a umidade da pele ${ }^{2}$

\section{Atividades desenvolvidas pelas enfermeiras}

Manutenção das vias aéreas pérveas. Aspiração das vias aéreas superiores; Desobstrução das vias aéreas; Lavagem da narinas com soro fisiológico; Orientar à higiene nasal e exercícios respiratórios. Manutenção das vias aéreas pérveas. Observar presença de obstáculos à passagem de ar; Aspiração das vias aéreas superiores; Desobstrução das vias aéreas; Lavagem da narinas com soro fisiológico; Orientar à higiene nasal e exercícios respiratórios.

Monitorização com oximetria de pulso; Colheita de exame para gasometria arterial.

Conforto físico e psicológico à criança;Tranqüilizar a criança e a mãe; Promover ambiente tranqüilo; Manter criança no colo da mãe.

Monitorização/verificação dos sinais vitais; Monitorização com oximetria de pulso.

$$
\text { Oxigenoterapia CPM. }
$$

Monitorização/ verificação de sinais vitais; Observação do padrão respiratório da criança.

Monitorização/ verificação de sinais vitais.

Monitorização/ verificação de sinais vitais.

Monitorização/ verificação de sinais vitais.Observação do padrão respiratório da criança.

Avaliação pulmonar (ausculta); Realizar exame físico.

Avaliação pulmonar (ausculta); Realizar exame físico.

Monitorização com oximetria de pulso.

Monitorização/ verificação de sinais vitais.

Monitorização com oximetria de pulso; Observar sinais e sintomas de choque; Manutenção das vias aéreas pérveas; Colheita de exame para gasometria 


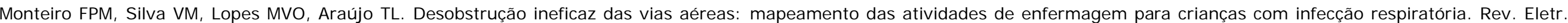
Enf. [Internet]. 2008; 10(3):563-72. Available from: http://www. fen.ufg. br/revista/v10/n3/v10n3a02.htm

\begin{tabular}{|c|c|}
\hline Atividades propostas pela NIC & Atividades desenvolvidas pelas enfermeiras \\
\hline & arterial. \\
\hline Monitorar a presença de cianose central e periférica ${ }^{2}$ & Avaliar a perfusão periférica; Colheita de exame para gasometria arterial. \\
\hline Observar o tipo e a quantidade de secreções obtidas ${ }^{3}$ & $\begin{array}{c}\text { Observar consistência, característica e quantidade de secreção; Observar presença } \\
\text { de secreções nasais e orais. }\end{array}$ \\
\hline Obedecer aos cinco direitos da administração de medicamentos ${ }^{4}$ & Administração de medicamentos CPM; \\
\hline $\begin{array}{c}\text { Documentar a administração do medicamento e a resposta da criança, } \\
\text { conforme as orientações da instituição }{ }^{4}\end{array}$ & Registro de enfermagem. \\
\hline Encorajar a criança a envolver-se nas trocas de posição, quando adequado ${ }^{5}$ & Incentivar à mudança de decúbito; \\
\hline $\begin{array}{c}\text { Monitorar o estado de oxigenação antes e depois da troca de posição, quando } \\
\text { adequado }^{5}\end{array}$ & Monitorização com oximetria de pulso; \\
\hline Elevar a cabeceira da cama, quando adequado ${ }^{5}$ & Elevar a cabeceira do leito; \\
\hline $\begin{array}{c}\text { Posicionar para facilitar a combinação entre a ventilação/perfusão, quando } \\
\text { adequado }^{5}\end{array}$ & $\begin{array}{c}\text { Favorecer a expansão torácica, com mudança de decúbito; Incentivar à mudança de } \\
\text { decúbito; }\end{array}$ \\
\hline Auxiliar com mudanças freqüentes de posição, quando adequado ${ }^{6}$ & Incentivar à mudança de decúbito. \\
\hline Posicionar para minimizar os esforços respiratórios ${ }^{6}$ & Encorajar à criança ao repouso no leito; Manter cabeceira elevada. \\
\hline $\begin{array}{c}\text { Monitorar a oxigenação e instituir medidas para promover a oxigenação } \\
\text { adequada dos órgãos vitais }\end{array}$ & $\begin{array}{l}\text { Monitorização com oximetria de pulso; Avaliar a perfusão periférica; Colheita de } \\
\text { exame para gasometria arterial. }\end{array}$ \\
\hline Monitorar a perfusão tissular, quando adequado ${ }^{7}$ & $\begin{array}{l}\text { Monitorização com oximetria de pulso; Avaliar a perfusão periférica; Colheita de } \\
\text { exame para gasometria arterial. }\end{array}$ \\
\hline Verificar a prescrição para a terapia $\mathrm{EV}^{8}$ & Administração de medicamentos CPM. \\
\hline Solicitar aos pais que segurem e conformem o filho, quando adequado ${ }^{8}$ & $\begin{array}{l}\text { Tranqüilizar a criança e a mãe; Promover ambiente tranqüilo; Conforto físico e } \\
\text { psicológico à criança. }\end{array}$ \\
\hline Oferecer apoio emocional, quando adequado ${ }^{8}$ & Tranqüilizar a criança e a mãe; Conforto físico e psicológico à criança. \\
\hline Selecionar uma veia adequada para a venopunção ${ }^{8}$ & Viabilizar acesso venoso. \\
\hline Inserir agulha conforme as instruções do fabricante ${ }^{8}$ & Viabilizar acesso venoso. \\
\hline Administrar terapia IV, conforme prescrição ${ }^{9}$ & Administração de medicamentos CPM;Administrar hidratação venosa CPM. \\
\hline Obter de 3 a 5 cc de amostra de sangue ${ }^{10}$ & Colheita de exame para gasometria arterial; \\
\hline $\begin{array}{c}\text { Permanecer com a criança e oferecer segurança e proteção durante períodos de } \\
\text { ansiedade }\end{array}$ & $\begin{array}{l}\text { Providenciar ambiente tranqüilo; Tranqüilizar mãe e criança; Promover conforto à } \\
\text { criança; Manter criança no colo da mãe; Conforto físico e psicológico à criança. }\end{array}$ \\
\hline
\end{tabular}

Intervenções NIC correspondentes - Sugeridas: 1 - Oxigenoterapia, 2 - Monitorização de sinais vitais, 3 - Aspiração de vias aéreas; 4 - Administração de

medicamentos: inalação; 5 - Posicionamento; 6 - Assistência ventilatória; 7 - Supervisão. Optativas: 8 - Inserção endovenosa EV; 9 - Controle de líquidos;

10 - Punção de vaso: amostra de sangue; 11 - Suporte emocional. 
Monteiro FPM, Silva VM, Lopes MVO, Araújo TL. Desobstrução ineficaz das vias aéreas: mapeamento das atividades de enfermagem para crianças com infecção respiratória. Rev. Eletr. Enf. [Internet]. 2008; 10(3):563-72. Available from: http://www.fen.ufg.br/revista/v10/n3/v10n3a02.htm.

Conforme demonstrado pela Tabela 1, pode-se observar que as atividades prioritárias da NIC são direcionadas às enfermeiras, que são responsáveis por ações como realizar fisioterapia respiratória, tapotagem e remoção de secreções. No entanto, ao correlacionar essas atividades, as enfermeiras assistenciais direcionam esse cuidado a outros profissionais, tais como fisioterapeutas. Nesse mesmo raciocínio, pode se destacar atividades prioritárias da NIC descritas de forma mais detalhada, enquanto as enfermeiras assistenciais apresentaram uma nomenclatura mais geral, menos descritiva, por exemplo, quando a atividade prioritária monitorizaçao respiratória, proposta pela NIC, menciona ações restritas ao exame físico pulmonar, como percutir o tórax anterior e posterior, dos ápices para as bases pulmonares, as enfermeiras assistenciais citam a realização do exame físico, não deixando claro as etapas envolvidas e a utilização dos métodos propedêuticos necessários para este fim.

$\mathrm{Na}$ Tabela 2, correspondente às intervenções sugeridas e optativas da NIC, observa-se que a maioria das atividades da NIC apresentou correspondência com várias outras citadas pelas enfermeiras. Entretanto, destacase que a nomenclatura utilizada pela NIC abrange ações mais gerais que incluem atividades mais específicas do que as citadas pelas enfermeiras. Dentre elas, pode-se destacar: manutenção das vias aéreas pérveas, aerossolterapia conforme prescrição médica (CPM), e elevar a cabeceira do leito, correspondendo às atividades NIC, respectivamente: manter vias aéreas desobstruídas, administrar tratamentos com aerossol conforme adequado e elevar a cabeceira da cama quando adequado.

Nesse sentido, verifica-se que, as enfermeiras utilizaram uma nomenclatura mais usual à prática da enfermagem brasileira, mantendo o mesmo significado e usando palavras diferentes.

Potter e Perry ${ }^{(12)}$ utilizam nas suas prescrições de enfermagem a nomenclatura "manutenção da permeabilidade da via aérea" quando se referem às ações de controle das vias aéreas desobstruídas. As atividades prescritas pelas enfermeiras assistenciais apresentavam-se pontuais e muitas delas se referiam a uma ou a várias atividades preconizadas pela NIC. Foram observadas, também, algumas ações prescritas pelas enfermeiras que necessitavam de um maior detalhamento e adequação, já que o estudo direcionava-se ao cuidado de crianças com até cinco anos de idade.

As atividades prescritas pelas enfermeiras necessitavam de um maior detalhamento e adequação, as quais se podem citar: estimular a tosse, estimular a respiração profunda e viabilizar acesso venoso. Enquanto as atividades preconizadas pela NIC especificam as ações de enfermagem no ensino, orientação da técnica, porém omitem a idade do(a) paciente. $\mathrm{Na}$ implementação do plano de cuidados prescrito a um cliente, a enfermeira deve ensinar o cliente a incorporar novos conhecimentos em relação à sua própria saúde ou ao controle de um distúrbio, como também auxiliá-lo a realizar atividades por si mesmo ${ }^{(13)}$.

As atividades NIC abrangem cuidados mais gerais os que dizem em respeito ao posicionamento da criança com problemas respiratórios ${ }^{(14)}$, por exemplo: posicionar a criança para maximizar o potencial ventilatório, correspondendo a diversas ações mais específicas prescritas pelas enfermeiras assistenciais: elevar a cabeceira do leito, favorecer a expansão torácica com mudança de decúbito e incentivar para mudança de decúbito. Em geral, percebeu-se que, quando as enfermeiras se referiam às atividades que favoreciam o conforto respiratório, como o alívio da dispnéia, sua caracterização era de uma situação emergencial, que necessitava de uma intervenção imediata para reversão do quadro clínico.

Em outro momento, também na Tabela 2, aparece a atividade da NIC obtenção de 3 a 5 cc de amostra de sangue, que as enfermeiras relacionaram como colheita de exame para gasometria. Ressalta-se que essas atividades foram direcionadas às crianças acometidas por patologias respiratórias, especificamente com desobstrução ineficaz das vias aéreas com até cinco anos de idade. Para esta clientela, a enfermeira deve implementar ações mais 
Monteiro FPM, Silva VM, Lopes MVO, Araújo TL. Desobstrução ineficaz das vias aéreas: mapeamento das atividades de enfermagem para crianças com infecção respiratória. Rev. Eletr. Enf. [Internet]. 2008; 10(3):563-72. Available from: http://www.fen.ufg. br/revista/v10/n3/v10n3a02.htm.

detalhadas que levem em consideração o processo de adoecer da criança e seus aspectos emocionais, na tentativa de minimizar o sofrimento ocasionado pela hospitalização e não apenas colher sangue sem ter um objetivo definido.

Além da existência de algumas atividades preconizadas pela NIC e listadas pelas enfermeiras assistenciais como tarefas que se diferenciavam somente pela nomenclatura utilizada, outras atividades descritas pela NIC foram consideradas não-correspondentes às prescritas pelas enfermeiras. Esse fato estava relacionado às ações que requeriam cuidados mais especializados, exigindo recursos não disponíveis pelo serviço de saúde, ações direcionadas ao cuidado da clientela com estado mais crítico de saúde, ou atividades que requeriam uma qualificação profissional específica.

Dentre as atividades não-correspondentes, destaca-se: usar técnicas lúdicas para encorajar a respiração profunda de crianças, por sua relevância no atendimento à criança com Desobstrução ineficaz de vias aéreas e pela importância de sua realização pela equipe de enfermagem, representando um cuidado preconizado pela literatura, porém algumas vezes esquecido na prática clínica. Técnicas lúdicas podem ser empregadas para crianças mais jovens acometidas por asma, visando a ampliar o tempo expiratório e a aumentar a pressão expiratória, as quais incluem soprar balões de algodão ou uma bola de pinguepongue sobre uma mesa, soprar um catavento ou bolhas, ou impedir que um papel caia ao soprá-lo contra a parede ${ }^{(15)}$.

A implementação de tarefas que utilizam o lúdico no cuidado à criança incentiva a participação da mesma no tratamento, como também contribuem para a melhora do estado de saúde ${ }^{(16)}$. Quando a assistência de enfermagem é dirigida à criança, a equipe de enfermagem deve estar consciente de que esta possui modos peculiares de sentir e perceber as adversidades do meio em que se encontra inserida e o profissional da saúde deve entender a linguagem infantil e implementar a atividade lúdica no âmbito hospitalar interpretando o real significado da mensagem ${ }^{(17)}$. Ressalta-se que atividades lúdicas são pouco realizadas, pois requerem mais tempo e qualificação da enfermeira, além da colaboração da criança. Por conta disso, essas atividades acabam sendo assumidas por outros profissionais e passam a ser negligenciadas pelas enfermeiras.

\section{CONCLUSÃO}

Foram listadas pelas enfermeiras assistenciais 48 atividades desenvolvidas no seu cotidiano referentes ao diagnóstico de enfermagem Desobstrução ineficaz de vias aéreas. Dentre as 327 atividades apresentadas nas 14 intervenções da NIC para o diagnóstico Desobstrução ineficaz das vias aéreas, 63 foram consideradas correspondentes após a análise do comparativo, diferenciando-se apenas pela nomenclatura utilizada na NIC. Algumas ações prescritas pelas enfermeiras necessitavam de um maior detalhamento e adequação, já que o estudo direcionava-se ao cuidado de crianças com até cinco anos de idade. Outras atividades não apresentaram correspondência por caracterizarem ações que dependiam de recursos especializados do serviço de saúde, ações direcionadas ao cuidado da clientela com estado crítico de saúde e atividades que requeriam uma qualificação profissional específica.

Diante dos resultados obtidos, ressalta-se que este trabalho poderá contribuir para a assistência de enfermagem às crianças jovens acometidas por problemas respiratórios e que apresentam o diagnóstico de enfermagem Desobstrução ineficaz de vias aéreas, como também proporcionar um cuidado específico e qualificado. Em relação à prática profissional, considera-se que 0 estabelecimento de intervenções prioritárias podem auxiliar as enfermeiras assistenciais a desenvolver planos de cuidados específicos e com maior poder de resolutividade. Ademais, para a organização do serviço, isto se reverte em ganho de tempo, maximização de recursos e diminuição de gastos com intervenções desnecessárias ou mesmo com baixa efetividade. Entretanto, é necessário considerar que o presente estudo foi desenvolvido numa realidade específica e com um grupo limitado de enfermeiras. Assim, é possível que outras atividades de enfermagem 
Monteiro FPM, Silva VM, Lopes MVO, Araújo TL. Desobstrução ineficaz das vias aéreas: mapeamento das atividades de enfermagem para crianças com infecção respiratória. Rev. Eletr. Enf. [Internet]. 2008; 10(3):563-72. Available from: http://www.fen.ufg. br/revista/v10/n3/v10n3a02.htm.

sejam identificadas em outras realidades para o mesmo diagnóstico através de uma amostra mais representativa. Por outro lado, convém ressaltar que é possível identificar outros locais com recursos semelhantes àqueles disponíveis na realidade onde o presente estudo foi desenvolvido.

\section{REFERÊNCI AS}

1. Facanha MC, Pinheiro AC. Doenças respiratórias agudas em serviços de saúde entre 1996 e 2001, Fortaleza, CE. Brasil. Rev Saud Publ. 2004;38(3):346-50.

2. Chiesa AM, Westphal MF, Akerman M. Doenças respiratórias agudas: um estudo das desigualdades em saúde. Cad. Saúde Pública. 2008;24(1):55-69.

3. Oliveira GF, Dantas FDC, Fonseca PN. O impacto da hospitalização em crianças de 1 a 5 anos de idade. Revista SBPH. 2004; 7(2): 37-54.

4. Gonçalves-Silva RMV, Valente JG, LemosSantos MGF, Rosely S. Tabagismo no domicílio e doença respiratória em crianças menores de cinco anos. Cad. Saúde Pública. 2006; 22(3): 579-86.

5. Secretaria Municipal de Saúde; Prefeitura Municipal de Fortaleza. Boletim de Saúde de Fortaleza: Doenças respiratórias agudas. Fortaleza (Brasil): Prefeitura Municipal de Fortaleza; 2002.

6. Nascimento LFC, Marcitelli R, Agostinho FS, Gimenes CS. Análise hierarquizada dos fatores de risco para pneumonia em crianças. J. Bras. Pneumol. 2004; 30(5): 445-51.

7. North American Nursing Diagnosis Association. Diagnósticos de Enfermagem da NANDA: Definições e classificações 2007-2008. Porto Alegre: Artmed; 2008.

8. Dochterman JC, Bulechek GM. Classificação das Intervenções de Enfermagem. 4a ed. Porto Alegre: Artmed; 2008.

9. Monteiro FPM, Silva VM, Lopes MVO. Diagnósticos de enfermagem identificados em crianças com infecção respiratória aguda. Revista Eletrônica de Enfermagem [Internet]. 2006 [cited 2006 dez 20];8(2):213-21. Available from: http://www.fen.ufg.br/revista/revista8_2/v8n2a 06.htm
10. Napoleão AA. Estudo da aplicabilidade de intervenções da NIC no atendimento a crianças com o diagnóstico de enfermagem Desobstrução Ineficaz das Vias Aéreas relacionado à presença de via aérea artificial em um centro de terapia intensiva pediátrico [tese]. [Ribeirão Preto]: Escola de enfermagem de Ribeirão Preto da Universidade de São Paulo; 2005.

11. Ministério da Saúde. Conselho Nacional de Saúde. Resolução no 196/96. Decreto no 93.933 de janeiro de 1987. Estabelece critérios sobre pesquisa envolvendo seres humanos. Bioética. 1996; 4 Suppl 2:15-25.

12. Potter PA, Perry AG. Fundamentos de enfermagem. 6a edição. Rio de Janeiro: Editora Elsevier, 2006.

13. Capernitto LJ . Diagnósticos de enfermagem: aplicação à prática clínica. 10ạ edição. Porto Alegre: Artmed; 2005.

14. Martins I. Nursing intervention analysis for the nursing diagnosis ineffective airway clearance. In: North American Nursing Diagnosis Association. NANDA, NIC, NOC 2004: Working Together for Quality Nursing Care: Striving Toward Harmonization. Chicago: NANDA I nternational; 2004. p. 107.

15. Hockenberry MJ, Wilson D, Winkelstein ML. Wong: fundamentos de enfermagem pediátrica. 7a ed. Rio de Janeiro: Mosby; 2006.

16. Napoleão AA. Ineffective airway clearance: applicability of NIC priority interventions in a Brazilian pediatric intensive care unit. Int Jf Nurs Terminol Classif. 2006; 17(1): 76.

17. Carvalho LS, Silva CA, Santos ACO, Camargo $\mathrm{CL}$. The child facing the stress beforesurgical. Exploratory study.. Online Braz J Nurs [Internet]. 2006 [cited 2007 fev 17];5(3). Available from: http://www.uff. br/objnursing/index. php/nursing /article/view/508/117.

Artigo recebido em 12.06.07

Aprovado para publicação em 30.09.08 